\title{
THE GENETICAL SOCIETY
}

\section{(Abstracts of papers presented at the TWO HUNDRED AND THIRTEENTH MEETING of the Society held from 3rd to 6th April 1990 at the UNIVERSITY OF BIRMINGHAM)}

\section{Human Biometrical Genetics since 1970}

\section{Lyndon Eaves}

Department of Human Genetics, Virginia

Commonwealth University, U.S.A.

In 1970 Jinks and Fulker published their seminal paper on the application of Biometrical Genetics to the analysis of human behaviour. The basic principles of model-building and model-fitting outlined in that article have now become firmly established in the mainstream of human quantitative genetics. The two decades since this paper have produced a wide range of theoretical and substantive contributions to the analysis of genetic and environmental contributions to human variation which, although they embody the basic principles set out by Jinks and Fulker, have taken the subject into new areas. This paper will outline some of the major theoretical developments and, where appropriate, illustrate them with new data. Among the issues to be discussed are: the inclusion of social interaction in models for family resemblance; analysing the mechanisms underlying mate selection; the contribution of nonadditive genetic effects; genotype-environment interaction; sex-differences in gene expression; the genetic control of development change and the timing of events; causal relationships among multiple variables. Some of the issues will be elucidated by consideration of data on human behaviour and cardiovascular risk factors.
Although the relationship between heredity and behaviour has been a constant theme in psychology since the time of Francis Galton, it was not until the 1960s that behaviour genetics established itself as a distinct field of inquiry in psychology. In 1960 Fuller and Thompson's textbook was published, and before the end of the decade, the Behaviour Genetics Association had been founded along with its journal, Behaviour Genetics, and strong research programs had emerged in Great Britain and the United States. A number of geneticists encouraged psychologists, offering much-needed methodological sophistication and enthusiastic support. In Great Britain John Jinks was the dominant influence among geneticists during this period, through fruitful collaboration with psychologist Peter Broadhurst and his research group, when the main focus was on research into the genetic control of animal behaviour. Between 1970 and 1980 the emphasis turned more to research into complex human traits. While the current emphasis in the field as we enter the 90 s is now more on social and biological processes in human and animal behaviour, John Jinks' influence is still in evidence, primarily as a matter of style among his former students and those they have trained. It was my good fortune as a beginning psychologist in 1964 to come under John's influence and to have been actively engaged in behaviour genetics ever since. I offer a personal view of the growth of the field during this period and of John Jinks' influence on it.

\section{Problems of polygene location with RFLPS}

\section{Mike Kearsey}

School of Biological Sciences, University of Birmingham, PO Box 363, Birmingham B15 2TT, England.
David W. Fulker

Institute of Behavioural Genetics, University of Colorado, U.S.A. 


\section{Chromosome segregation during meiosis in Saccharomyces cerevisiae}

\section{Giora Simchen}

Department of Genetics, The Hebrew University, Jerusalem 91904, Israel.

Normal meiosis consists of two consecutive nuclear divisions in which all the chromosomes behave in a concerted manner. Several yeast mutants may be directed through single-division meioses, yielding dyads of diploid spores (instead of tetrads of haploid spores). In these meioses, some chromosomes in each cell segregate reductionally, whereas others, in the same cell, segregate equationally. The choice between the two types of segregation is made individually by each chromosome pair, probably by the centromeric regions. Centromere substitutions on a given chromosome alter its segregation tendency, according to the tendency of the new centromere. When two homologous chromosomes carry different centromeres, they frequently segregate in a non-coordinated fashion, resulting in non-disjunction-like products.

The regular disjunction of the two homologous chromosomes in meiosis I also depends on their earlier pairing during zygotene-pachytene. Unpaired chromosomes commonly undergo meiotic non-disjunction. We are exploring various causes for non-disjunction, such as the presence of plasmids or extra chromosomes which compete for meiotic pairing with the major chromosomes. Conversely, deletion of a chromosomal region, which has an important role in pairing, may also increase the rate of meiotic non-disjunction. Thus we hope to define and characterise pairing sites in S. cerevisiae.

\section{Retrobiology: from yeast to HIV}

\section{Sue Kingsman}

Department of Biochemistry, University of Oxford.

\section{Effect of selection for isozyme} markers on quantitative traits in a double haploid population of barley

\author{
Wayne Powell, R. P. Ellis, B. P. Fisher and \\ D. Cawston
}

Scottish Crop Research Institute, Invergowrie, Dundee DD2 5DA, Scotland.

\section{Cross prediction in Nicotiana rustica}

\section{Harpal Pooni}

School of Biological Sciences, University of

Birmingham, PO Box 363, Birmingham B15 2TT, England.

\section{Biometrical genetics in plant breeding: evaluation of breeding strategy}

\section{J. W. Snape, C. N. Law and A. J. Worland \\ Cambridge Laboratory, Institute of Plant Science Research, Colney Lane, Norwich, U.K.}

Many of the characters which the plant breeder seeks to influence display "continuous variation" in segregating generations, where discrete genotypic classes are absent and there is a gradual quantitative change between extreme phenotypes. The science of Biometrical Genetics has been developed to analyse such variation. Undoubtedly the "Birmingham School" under the leadership of Professor Sir Kenneth Mather and Professor John Jinks has been the worlds' leading laboratory in the development and application of this methodology to plant breeding.

The methods of biometrical genetics provide both descriptive and predictive tools in plant breeding. As descriptive tools they allow the consequences of empirical plant breeding practice to be defined genetically. This enables alternative techniques to be evaluated theoretically and tested practically, thereby allowing objective criteria for their use to be formulated.

Nowhere has the utility of these methodologies been demonstrated better than in studies of cereals, particularly wheat and barley. Over the last three decades, for example, plant breeders and geneticists at the Plant Breeding Institute and its successor, the Cambridge Laboratory, IPSR, have extensively applied the techniques to help define breeding strategy and to elucidate the genetical control of economically important characters. The present paper will discuss some of these studies to illustrate the utility of the methodology using examples from work defining the consequences of methods of rapid generation advancement (single seed descent and doubled haploid procedures); and in evaluating the genetical basis of heterosis in wheat.

The use of techniques of molecular biology to map plant chromosomes is now leading towards 
an integration of biometrical genetics and "classical" genetics. The future prospects for using these techniques to locate and understand the individual genes mediating continuous variation will be illustrated and discussed with reference to our work on wheat.

\section{Applications of biometrical genetics for forage brassica breeding}

\section{J. E. Bradshaw}

Scottish Crop Research Institute, Invergowrie, Dundee DD2 5DA, Scotland.

\section{Biometrical genetics in plant breeding: theory and practice of prediction}

\section{P. D. S. Caligari}

Department of Agricultural Botany, School of Plant Sciences, University of Reading, Whiteknights, P.O. Box 221, Reading, RG6 2AS, U.K.

The practice of plant breeding goes back to when primitive Man first started to sow seeds and have a settled form of agriculture. At this time and for much of our history the cycle of simply picking 'types' that did better, preferentially sowing these in the next season, making speculative cross-pollinations, etc. marked plant breeding as an art rather than being based on science.

The basis for change in our approach was laid down with Mendel in 1900 . Breeders quickly saw the potential of the new science of "Genetics" as giving them some prediction of the outcome of their actions in terms, at least, of characters which we now recognize as being controlled by major genes. This was a considerable step forward but still left most of the variation in characters inexplicably determined and of unpredictable behaviour because the effects of individual genes could not be recognized. The work of a number of more mathematically able scientists, particularly R. A. Fisher, laid the foundations for the analysis and understanding of this variation which is often termed "continuous" and the development of a branch of genetics called "Biometrical" or "Quantitative". This branch of the subject developed several arms and one of the most significant of these for plants "grew" in Birmingham, especially under the leadership of Professor Sir Kenneth Mather. A significant part of this "lineage" was the late Professor J. L. Jinks who made a major and wide ranging contribution to the subject of Biometrical Genetics.

I will present and discuss some results which reflect a small part of John Jinks' contribution and which led to a set of techniques of importance in applied as well fundamental studies. These are techniques that have particularly and directly extended the ability of breeders to predict, in a very powerful way, the range of genotypes obtainable from a cross. The techniques have been collectively termed "Cross Prediction" and they are being extensively examined, and in some cases implemented, with some enthusiasm by plant breeders. Evidence presented shows them to have the potential to radically affect plant breeding strategies and clearly mark it as a "science" rather than an "art".

\section{Cross prediction in spring barley breeding}

\author{
Bill Thomas \\ Scottish Crop Research Institute, Invergowrie, \\ Dundee, DD2 5DA, Scotland.
}

\section{DNA independent mRNA amplification is prerequisite for the killing of plasmid free segregants by the hok/sok system of plasmid R1}

\section{Ken Gerdes, Thomas Thisted and Jan Martinussen \\ Dept. of Molecular Biology, Odense University, Campusvej 55, DK-5230 Odense M, Denmark.}

Bacterial plasmids code for several types of maintenance functions, such as partitioning functions, cell division inhibition functions, and site-specific resolution functions. Presumably, all these functions act in concert, thereby assuring that each new daughter cell receives at least one plasmid molecule at cell division. Recently, we have identified yet another type of plasmid maintenance function, the hok/sok system from plasmid R1 (homologous to $\mathrm{flm}$ of $\mathrm{F}$ ). The hok/sok system mediates plasmid stability via killing of plasmid free segregants. The effector of the system is a toxic protein, Hok (host killing), which is synthesised in the plasmid free segregants only. Expression of the hok gene is regulated by the sok gene product, a small antisense RNA complementary to the hok 
mRNA leader region. We have observed, that the hok mRNA is extremely stable, whereas the sok antisense RNA is extremely labile. We suggest, that this differential RNA decay causes the plasmid free segregants to experience a rapid decay of the repressor RNA. This, in turn, causes depression of translation of the hok mRNA and synthesis of the Hok protein. Obviously, such a mechanism would lead to a rapid and selective killing of the newborn plasmid free cells.

One main theme of this simple model has now been verified, namely that the differential decay of the hok and the sok RNAs is the basis for the expression of the Hok protein in the new plasmid free cells. Surprisingly, an additional control loop seems to be superimposed on the regulatory scheme described above. Several independent lines of evidence now suggest that the hok mRNA is amplified in situations where the sok antisense RNA is not present. Most importantly, the amplification also seems to occur in plasmid free cells devoid of the hok gene. Experimentally, we have observed the synthesis of the new mRNA species, denoted the phantom hok mRNA. The phantom mRNA is 70 nucleotides shorter than the wild type hok mRNA, but encompasses the hok gene coding frame. Point-mutations in the hok/sok system, which abolish the synthesis of the phantom mRNA, also eliminates the killing of plasmid free cells. Thus, the effector mRNA of the system seems to be the phantom mRNA and not the hok mRNA.

Most controversial, the synthesis of the phantom mRNA seems to occur independently of the DNA template. Rather, the synthesis seems to be dependent on an intact (stable) hok mRNA. We are therefore tempted to suggest, that the hok mRNA is template for the synthesis of the phantom mRNA, and that this synthesis is regulated by the sok antisense RNA. The biochemical events leading to synthesis of the phantom mRNA is not known.

\section{Plasmid (in)stability in Bacillus subtilis}

\section{Sierd Bron, Peter Haima, Ben Peeters, Ad Peijnenburg, Linda Oskam and Siger Holsappel}

Department of Genetics, Center of Biological Sciences, Kerklaan 30, 9751 NN Haren, The Netherlands.

The use of $B$. subtilis and other bacilli as efficient hosts for molecular cloning has been hampered for at least two reasons: (i), instability of recombinant plasmids, and (ii), low efficiences in (shotgun) cloning.

Major breakthroughs in the understanding of many of these problems have recently been attained. These will be discussed. A key observation was that the small plasmid vectors used for cloning in B. subtilis replicate via single-stranded (ss) DNA intermediates (Te Riele, H., et al., 1986: PNAS 83, 2541; Gruss, A., and Ehrlich, S. D., 1989: Microb. Reviews 52, 231), most likely via rolling-circle replication ( $\mathrm{RCR}$ ). This mode of replication has serious consequences both for plasmid maintenance (segregational stability) and for plasmid rearrangements, most frequently deletions (structural stability). In particular, the frequently used plasmids from Staphylococcus aureus show low stabilities.

We study segregational plasmid stability with two model plasmids: pUB110 (from $S$. aureus) and pTA1060 (from $B$. subtilis). The first plasmid shows a strong size-dependent instability (Bron, S., and Luxen, E., 1985: Plasmid 14, 235; Bron, S., et al., 1988: Plasmid 19, 231), which explains part of its poor cloning properties. The second plasmid is very stable and is an excellent cloning vector. Both plasmids contain a function, not being part of the minimal replicon, which is required for optimal segregational stability (Bron, S., et al., 1988: Plasmid 19, 231; Bron, S., et al., 1987: Plasmid 18, 8). These functions appeared to be the initiation sites for lagging strand synthesis during RCR.

Structural stability was studied with two model systems. In the first synthetic oligonucleotides were used to create direct repeats (DRs) of various lengths. The frequency of deletion between the repeats was measured by a positive selection system (Peeters, B. P. H., et al., 1988: Mol. Gen. Genet. $212,450)$. The length of the DRs and the presence of flanking inverted repeats stimulate this process. $\mathrm{RCR}$, generating ssDNA, stimulates this process greatly.

In the second model system random deletions were selected in a pen $\mathrm{P} /$ lac $\mathrm{Z}$ fusion, using the blue/white colony test on X-gal plates. Deletions in this system did not appear to be affected by $\mathrm{RCR}$. The results rather suggest that topoisomerase I, possibly in conjunction with active transcription, is involved in the generation of these deletions (Peijnenburg, A. A. C. M., et al., 1988: Plasmid 20, 23).

Based on the stable plasmid pTA1060, we have developed a series of highly efficient and versatile cloning systems for B. subtilis (Haima, P., et al., 1987: Mol. Gen. Genet. 209, 335; Haima, P., et al., 
to be published). These systems enable: (1), the stable cloning of large inserts $(>30 \mathrm{~kb}) ;(2)$, the cloning of several genes which are difficult to clone in $E$. coli; (3), direct clone selection in a $\beta$-galactosidase $\alpha$-complementation system; (4), forced cloning and transformation with plasmid monomers in a plasmid marker rescue system.

\section{Molecular characterization of the partitioning system of plasmid RP4}

\section{Helmut Schwab}

Institut für Biotechnologie, Schoegelgaase 9, Graz, Austria.

\section{Plasmid maintenance in chemostat cultures of enteric bacteria}

\section{Jeff Cole}

School of Biochemistry, University of Birmingham, Birmingham B15 2TT, U.K.

It is now possible to clone virtually any gene into a plasmid vector and express it for the formation of a potentially useful product in a convenient host. Nevertheless, problems still arise during process scale-up because the presence of a plasmid imposes an unnatural burden on the host cell, a burden which could be relieved if only the plasmid were lost as a result of spontaneous mutation.

Two main aims dominate the various strategies employed in the design of cloning vectors. The first aim is to minimise the frequency of plasmid loss by segregational instability, or changes in plasmid structure by transportation, recombination or error prone replication events. The second is to minimise the stress imposed on the host cell during growth due to expression of the cloned gene, high plasmid copy numbers or the presence of multiple replication origins. Even when all such strategies are combined to optimise the genetic stability of both the plasmid and its host, it is impossible to eliminate the residual frequency of spontaneous plasmid loss due to mutations either in the plasmid itself or in the host chromosome. Nevertheless, there remain additional strategies that can be employed to optimise the yield of the desired product on an industrial scale.

Once a microbial culture containing a recombinant plasmid becomes contaminated with a subpopulation that is either plasmid free or unable to express the desired product, the rate at which the unwanted population accumulates depends primarily on two factors. These are the rate of generation of further variants by repeated mutation events, and the growth advantage of the variant populations relative to the initial strain. The host, its growth rate and the composition of the growth medium can all be varied in attempts to minimise any disadvantage of the required production strain. Results of experiments with laboratory scale chemostat cultures of Escherichia coli HB101 [pAT153] have provided specific examples of how such strategies work in practice. They include the demonstration of the beneficial effects on plasmid retention of increasing the growth rate, changing the growth-limiting nutrient from carbon to nitrogen, phosphorus or sulphur, or avoiding a functional tetracycline-resistance determinant in the cloning vector. Attempts to isolate fermenterexperienced hosts which are better able to retain the original plasmid will also be reviewed.

\section{DNA stability in Streptomyces}

John Cullum, Christine Dein and Waldo Rörig LB Genetik, Universität Kaiserslautern, Paul-EhrlichStr. 22, D-6750 Kaiserslautern, F.R.G.

Analysis of plasmid stability in Streptomyces is complicated by the questions of segregation during mycelial growth and spore formation. In some cases, differences in product formation between different recombinant plasmids can be attributed to the effects of segregational instability. In other cases, structural instability of plasmids can be a problem. The low copy number plasmid SPC2* contains a "stability region", whose presence reduces the frequency of segregational instability; we are using "stability-probe" vectors to analyse these functions.

Streptomyces species are especially prone to genetic instabilities in which large chromosomal deletions (often longer than $500 \mathrm{~kb}$ ) and DNA amplifications occur. In some cases the instability is stimulated by the presence of plasmids. DNA amplification of chromosomally integrated cloned genes promises the possibility of obtaining high copy number stable clones. 


\section{Selfish DNAs and traumatic transformations in the genetic manipulation of yeast}

\section{S. G. Oliver}

Manchester Biotechnology Centre, University of Manchester Institute of Science and Technology, PO Box 88, Manchester M60 1OD.

The budding yeast Saccharomyces cerevisiae is an organism which is very amenable to genetic manipulation using recombinant DNA techniques. Systems are available for DNA-mediated transformation and the organism's stringent requirement for homology in genetic recombination may be exploited in order to replace a wild-type copy of a gene on a chromosome with a mutant copy produced in vitro. By these techniques, very specific alternations may be made to the yeast genome. Our confidence in this specificity is dependent on the assumption that transformation is a neutral process which produces no other changes in the organism's genotype or phenotype. In this talk, this assumption will be questioned.

A series of experiments will be presented which examine the stability of the native $2 \mu$ plasmid in yeast populations. Evidence which demonstrates that this plasmid is a selfish DNA molecule which makes no positive, or a small negative, contribution to its host's fitness will be shown. It will be explained that cells which have lost the $2 \mu$ plasmid spontaneously exhibit a different phenotype from those which have been cured of the molecule by a process involving DNA-mediated transformation. From this, it will be argued that the cured cells have been genetically altered as a direct result of the transformation process. Evidence in support of this contention will be presented.

\section{Physiological aspects of growth and stability in yeast}

\section{Anthony Mason}

Swiss Federal Institute for Water Pollution Control (EAWAG). Swiss Federal Institutes of Technology Zürich, Ueberlandstrasse 133, CH-8600 Dübendorf, Switzerland.

In the past few years, there has been increasing interest in the use of microorganisms for the pro- duction of heterologous polypeptides and proteins. The main advantages associated with the use of yeasts as a host for the production of eukaryotic heterologous proteins are well known, and several systems have been developed for the synthesis of various pharmaceutical and fine chemical products such as hepatitis B surface antigen, interferons, calf chymosin etc. Optimization of production systems and large scale industrial application of recombinant DNA mediated systems, requires evaluation and maximization of both the genetic and physiological potentials available. This sets as a prerequisite the detailed understanding of the behaviour of all elements of the system, their interactions and the consequences of both internally and externally mediated perturbations, and of long term physical, chemical and biochemical change. Genetical and physiological optimization are closely linked and system performance has to be evaluated in terms of each. With respect to the question of stability of genetically manipulated strains, in theory it is not possible to consider genetic stability without considering the physiology and similarly one cannot study physiology or physiological stability without considering the genetics. In practice the situation very often looks somewhat different. There has been a great deal of very good qualitative research on the genetic aspects of stability in recombinant systems, especially in the case of the bacteria. In yeasts, despite the tremendous potential that these systems have to offer, the level of understanding still lags significantly behind that of the bacteria and whilst many phenomenological effects have been described, the genetic and physiological basis for these remain largely unresolved.

In the absence of recombinant DNA in microorganisms, physiological aspects of growth are highly complex. The presence of additional DNA interacting directly or indirectly with the biosynthetic, degradative, energetic and secretory pathways and mechanisms in the host cell is likely to have an adverse effect on physiological efficiency and thereby favour the production, via destabilization of the original genetic modification or by some related mechanism, of non-functional or ineffective phenotypes in a desired recombinant process construction. Since the physiology of yeasts used as hosts for recombinant DNA in both fundamental and applied situations is largely governed by the conditions under which it is cultivated, then the stability of the system has to be understood from this perspective and it is the purpose of this contribution to try and elucidate what is currently known of this and what remains to be understood. 


\section{Genetic stability in large scale fermentation of a transformed yeast strain producing $\mathrm{HBs} A \mathrm{G}$}

\author{
Nigel Harford \\ Smith Kline Biologicals, B1330 Rixensart, Belgium.
}

\section{Genetic analysis of chromosome stability in Saccharomyces cerevisiae}

\section{Stephen Kearsey, Cathryn Tambini and David Kipling \\ Department of Zoology, South Parks Road, Oxford, OX1 3PS.}

Yeast chromosomes require both origins of replication (autonomously replicating sequences, ARS) and a centromere for high mitotic stability. We have isolated mutations that enhance the mitotic stability of replication-defective minichromosomes, and these mutations are of two types: (i) cis-acting sequence changes that generate new replication origins; (ii) trans-acting mutations that identify genes whose products can affect replication initiation.

The cis-acting mutations were obtained in a plasmid originally lacking an ARS. Analysis of ten independent mutants shows that in two cases a single nucleotide change in the prokaryotic vector DNA creates an ARS, whereas in six other cases small duplications of vector DNA are responsible. This de novo generation of eukaryotic replication origins in prokaryotic DNA suggests that the requirements for initiation are rather simple.

We have recently characterized two genes that can affect ARS function. Change of function mutations in the RAR3 gene can enhance ARS function, and we have evidence that the RAR3 protein functions as a transcription factor. In contrast, null mutations in the RAR5 gene stimulate ARS function implying that the product can act as a repressor of initiation.

\section{Progress on gene manipulation and genetic stability research in filamentous fungi}

\section{Cees A. M. J. J. van del Hondel \\ TNO Medical Biological Laboratory, P.O. Box 45, 2280 AA Rijswijk, The Netherlands.}

During the last few years, a number of genetransfer systems, based on auxotrophic and dominant selectable markers, have been developed to study gene expression and gene regulation in filamentous fungi. With these systems it is now possible to carry out genetic manipulation experiments which are necessary for molecular genetic analysis of various biological processes occurring in filamentous fungi. This means that strategies and vectors were developed and tested to perform, for instance, gene disruption, gene replacement, gene targeting and gene cloning experiments as well as for gene expression analysis. In addition, suitable vectors are available for selection and/or identification of strains which have integrated multiple copies of these vectors into their genome. For the construction of strains which overproduce a given protein, amplification of the gene coding for this protein can be achieved by using these vectors.

Up till now a limited number of studies were carried out to analyze the genetic stability of strains which contain one or more copies of vector DNA integrated in their genome. These studies have indicated that in Aspergillus species those vector molecules are relatively stable during mitosis. In other filamentous fungi sometimes less stability is observed. During meiosis, however, instability is generally observed. To date, almost no data is available about the genetic stability under fermentation conditions of strains which overproduce a certain protein, due to the presence of a high copy number of the gene coding for this protein.

During the lecture the state of the art of the present research on gene manipulation and genetic stability will be presented.

\section{Current methods for achieving stable integration and efficient expression of cloned genes in animal cells}

\section{G. T. Yarranton \\ Division of Molecular Genetics, Celltech Ltd, Slough, Berks.}

The use of mammalian cells as hosts in the expression of complex eukaryotic proteins is now widely employed. Efficient gene expression from single copy transfected genes is rare and often gene amplification is used to select for increased gene copy number and hence, protein expression. Although transfected genes are chromosomally integrated and toxic drug (e.g. methotrexate) selection is employed there is a conflict between cell 
growth and product yield and this can lead to the overgrowth of non-expressing variants.

I will describe the use of the glutamine synthetase dominant selectable marker gene in gene amplification based mammalian expression systems and the expression of several eukaryotic proteins. In addition I will describe the use of nonamplifiable expression systems and how these can be applied to the production of recombinant antibody genes. The use of antibody genes is particularly interesting in the study of expression and genetic stability, since production of a functional protein requires the assembly of distinct heavy and light chains. Over expression of the heavy chain gene or loss of light chain gene expression can lead to cell death whereas heavy chain loss is tolerated.

\section{Stability of T-DNA inserts and their expression in plants}

\section{G. Gheysen, P. Breyne, A. Müller*, A. Depicker, and M. Van Montagu}

Laboratorium voor Genetica, Rijksuniversiteit Gent, $B$-9000 (Belgium) and *Zentralinstitut für Kulturpflanzenforschung der DDR, DDR-4325 Gatersleben, D.D.R.

Agrobacterium tumefaciens is an efficient system for transferring foreign genes, located on the $\mathrm{T}$ DNA, into higher plant cells. The T-DNA in such transformed plant cells is usually present in one or a few copies that appear to be inserted at random in the plant genome. The majority of the regenerated plants have been shown to transmit their newly acquired gene(s) in a Mendelian fashion (Budar, F., et al., 1986: Genetics 114, 303; Chyi, Y. S. et al. 1986: Molec. Gen. Genet. 204, 64; De Block, M. et al. 1984: EMBO J. 3, 1681; Deroles, S. C. and Gardner, R. C. 1988: Plant Mol. Biol. 11, 365; Müller, A. J. et al. 1987; Mol. Gen. Genet. 207, 171; Sukhapinda, K. et al. 1987: Mol. Gen. Genet. 206, 491).

Several lines of transgenic Nicotiana tabacum plants containing a single locus with a chimeric neomycin phosphotransferase II (nptII) gene have been investigated over three generations (Müller, A. J. et al. 1987: Mol. Gen. Genet. 207, 171; unpublished results). Expression of the nptII during seed germination allows us to obtain segregation data from self-fertilized and back-crossed progenies by a simple seedling test. In this way, the meiotic loss rate of the kanamycin resistance was determined to be $10^{-3}$ to $10^{-4}$ (Müller, A. J. et al. 1987: Mol. Gen. Genet. 207, 171; unpublished results.)

Among the non-Mendelian ratios obtained, the most common are plants that produce no or less than expected kanamycin-resistant progenies (Budar, F., et al., 1986: Genetics 114, 303; Chyi, Y. S. et al. 1986: Mol. Gen. Genet. 204, 64; Deroler, S. C. et al. 1988: Plant Mol. Biol. 11, 365, Sukhapinda, K. et al. 1987: Mol. Gen. Genet. 206, 491; unpublished results). Hybridization studies suggest a loose association between high (three or more) T-DNA copy number and abnormal inheritance of the kanamycin resistance (Deroles, S. C., and Gardner, R. C. 1988: Plant Mol. Biol. 11, 365). There is also a correlation between plants showing reduced expression of the kanamycin resistance phenotype and plants that show low or no transmission of that phenotype to their progeny. However, there are exceptions in both cases.

The fact that poorly expressing plants tend to show reduced transmission ratios suggests that low transmission ratios suggests that low transmission is the result of low expression of the gene, rather than from rearrangement or deletion of the kanamycin resistance gene during meiosis. However, some plants express the resistance phenotype well but fail to transmit it to the progeny and others with no expression of the gene manage to transmit kanamycin resistance with Mendelian ratios. Differences observed between the expression and transmission data could be accounted for by the phenomenon that the npt-II gene is being shut down in some sectors of the plant, for example by methylation. Switching off the expression during plant development would also provide an explanation for the variable inheritance ratios sometimes observed between different seed capsules (Deroles, S. C. and Gardner, R. C. 1988. Plant Mol. Biol. 11, 365; unpublished results).

Hybridization analysis of T-DNA structure in transgenic plants and their progeny revealed that the T-DNA remains stable during vegetative proliferation and during sexual transmission. Exceptionally, genetic instability of the T-DNA has been observed (Chyi, Y. S. et al. 1986: Mol. Gen. Genet. 204, 64).

In conclusion, the T-DNA can be transmitted to progeny with a high degree of meiotic stability required for commercial applications. Instability of the T-DNA-encoded phenotype has been observed and is probably caused by epigenetic mechanism or, exceptionally, is correlated with rearrangements of an unstable T-DNA locus. 
24. Stability of centromeric

plasmids in haploid and

diploid yeast

V. Kumar, S. Keränen and J. Knowles

$V T T / B I O$, Tietotiez 2, Espoo 02150, Finland.

\section{Development of a}

transformation system for

Trichoderma reesei based on the

PYR gene of $N$. crassa and

stability of transformants

Franz Grubver and C. P. Kubicek

Institute of BiochemicalTechnology, Getreidemarkt9,

A-1060 Vienna, Austria.

\section{Stability of Penicillium}

chrysogenum transformants

Ernst Friedlin

Biochemie GmbH, A-6250 Kundl, Austria.

27. Genetic transformation in Schizosaccharomyces japonicus
A. Weber and M. Sipiczki
Department of genetics, L. K. University,
H-4010 Debrecen, PO Box 56, Hungary.

28. Stability of runaway replication plasmids in E. coli during continuous culturing

K. B. Strudsholm

Institute of Biotechnology, Block 223, D+H, DK-2800, Lyngby, Denmark.

29. Metabolic plasmid stability in immobilized recombinant yeasts

R. Laub

Unite de Biotechnologie, IIF-IMC-Ceria, 1 ,

Av. E. Gryzon, 1070 Bruxelles, Belgium.
30. Stability of recombinant

Penicillium chrysogenum

D. V. Renno

Dept. of Biotechnology, Polytechnic of Central London, 115 New Cavendish Street, London W1M 8JS.

\section{Stability and adaptations of} host/plasmid systems in continuous sulture

P. J. McDermott, P. Gowland, T. S. Gross and P. C. Gowland

Department of Biological Sciences, John Dalton Building, Chester Street, Manchester M1 5GD.

32. Plasmid maintenance and threonine expression in chemostat cultures of $E$. coli

Clara B. Nudel, B. Vosman, M. J. Teixeira de Mattos, O. M. Neijssel

Department of Microbiology, University of Amsderdam, The Netherlands.

33. Scaling up heterologous-geneproducts biosynthesis in E. coli: stability and physiology

A. Chmiel, A. Plucienniczak and C. Cierniewski Microbiology and Virology Centre, Polish Academy of Sciences, Piotrkowska 173, 90-477 Lodz, Poland.

34. Stability of recombinant plasmids in brewing yeast

Dina Vakeria and Edward Hinchliffe Research Department, Bass Brewing Ltd., 137 High Street, Burton-on-Trent, U.K.

35. A highly stable excretion vector for fermentation of $E$. coli

Diogo S. Ardaillon, M. D. Jensen, J.-L. Vribelarrea and Jean-Michel Masson Laboratoire de Genie Biochemique et Alimentaire, VA 544 du CNRS Institut National des Sciences Appliquees, Toulouse, France. 
36. Amplifying DNA sequences of Streptomyces zimosus

\section{Danilenko and L. Storadubceseor \\ Moscow National Research Institute of Antiobiotics, Norgatinskaya Str 3A, Moscow 113105, U.S.S.R.}

\section{Stability of TOL plasmids in} strains of Pseudomonas putida

\section{Arney and T. Keshavarz}

Department of Biotechnology, Polytechnic of Central London, 115 New Cavendish Street, London W1M 8JS.

\section{Stability of Biosynthetic plasmid pUKC112 in yeast MD4010d [pUKC112].}

\section{S. Patel and T. Keshavarz}

Department of Biotechnology, Polytechnic of Central London, 115 New Cavendish Street, London W1M 8JS.

39. Investigation of plasmid stability in immobilised recombinant $E$. coli using two-stage continuous culture systems

J. N. Barbotin, S. Sayadi, M. Nasri, F. Berry, J. Huang, C. Briasco and D. Thomas Laboratoire de Technologie Enzymatique, URA 523 du CNRS, Université de Technologie de Compiegne, BP649 60206 .

40. Characterization of the naturally temperature sensitive Streptomyces ghananensis plasmid PSG5.

W. Wohlleben et al.

Fakultät für Biologie, Universität Bielefeld,

Postfach 8640, 4800 Bielefeld 1, F.R.G.

41. Properties of plasmid vectors for cloning into Corynebacterium glutamicum

\section{A. Pühler et al.}

Fakultät für Biologie, Universität Bielfeld,

Postfach 8640, 4800 Bielefeld 1, F.R.G.
42. DNA independent mRNA amplification is prerequisite for the killing of plasmid free segregants by the hok/sok locus of plasmid R1

Thomas Thisted, Jan Martinussen and Ken Gerdes

Department of Molecular Biology, Odense University, 5230 Odense M., Denmark.

\section{Segregational plasmid}

(in)stability and molecular cloning in Bacillus subtilis

Sierd Bron, Siger Holsappel, Linda Oskam, Niels van der Lelie and Peter Haima Department of Genetics, Center of Biological Sciences, Kerklaan 30, 9751 NN Haren, The Netherlands.

\section{Structural plasmid (in)stability in Bacillus subtilis}

Sierd Bron, Siger Holsappel, Ad Peijen, Purification Relano and Ben Peeters

Department of Genetics, Center of Biological Sciences, Kerklaan 30, 9751 NN Haren, The Netherlands.

45. Minus origins and mobilization properties of the streptococcal plasmid pMV158

Daniel van der Lelie, Wilfried J. J. Meijer, Linda Oskam and Sierd Bron

Department of Genetics, Centre of Biological Sciences, Kerklaan 30, 9751 NN Haren, The Netherlands.

46. Efficient cloning systems for $B$. subtilis using $\beta$-galactosidase $\alpha$-complementation

Peter Haima, Douwe van Sinderen, Hans Schotting, Gerard Venema and Sierd Bron

Department of Genetics, University of Groningen, Biologisch Centrum, Kerklaan 30, 9750 AA Haren, The Netherlands. 
47. Investigation of the potential of a cryptic plasmid of Bacilus subtilis as a vector for cloned genes

Andrew P. Gleave ${ }^{1,2}$, Andy Mountain ${ }^{2}$ and Christopher M. Thomas ${ }^{1}$

${ }^{1}$ School of Biological Sciences, University of Birmingham, PO Box 363, Birmingham B15 2TT, UK and ${ }^{2}$ Celltech Ltd, 216 Bath Road,

Slough SL1 4EN, U.K.

\section{Towards an understanding of partitioning of bacterial plasmids}

Christopher M. Thomas', Mostaffa MotallebiVeshareh', Stuart Austin ${ }^{2}$ and Michael Davis ${ }^{2}$

${ }^{1}$ School of Biological Sciences, University of Birmingham, PO Box 363, Birmingham B15 2TT, U.K. and ${ }^{2} \mathrm{NCl}$-Frederick Cancer Research Center, PO Box B, Building 539, Frederick, Maryland 21701, U.S.A.

\section{Plasmid stability in}

Streptomyces lividans batch fermentation

C. A. Wrigley-Jones, H. Richards, C. Thomas, M. Lilly and J. Ward

Department of Chemical and Biochemical Engineering, University College, London, Torrington Place, London WC1E 7JE.

50. Regulatory cassette allowing efficient gene expression in the presence of aromatic hydro-carbons

S. Keil, I. Rowell, A. Al-Mayhrabi and H. Keil

Department of Biology and Biochemistry,

Brunel University, Uxbridge, Middlesex.

51. S-locus and related DNA sequences in Brassica oleracea

T. K. Bradshaw and C. G. Mousley

Shell Research Ltd., Sittingbourne Research Centre, Sittingbourne, Kent ME9 8AG.
52. Exonuclease III is essential for stability of pBR322 and pACYC184 in Escherichia coli

Ramsey James McFarlane

Department of Botany and Microbiology,

The University of Liverpool, PO Box 147,

Liverpool L69 3BX.

\section{Plasmid stability in}

Pseudomonas in culture and in soil related systems

E. Smit, E. Caldenhouen and J. D. van Elsas

Research Institute ITAL, P.O. 48, Wageningen, The Netherlands.

54. Elements involved in vector stability in Kluyveromyces lactis transformants

Michele Maria Bianchi and Laura Frontali Department of Cell and Developmental Biology, University of Rome, Piaza A. Moro 5, 00185 Roma, Italy.

55. Identification of a resolvase gene which imparts segregational stability on pAMB1-based vectors

T. J. Swinfield ${ }^{1}$, L. Janniere ${ }^{2}$, F. D. Ehrlich ${ }^{2}$ and N. P. Minton'

${ }^{1}$ Division of Biotechnology, PHLS Cama, Porton Down, Salisbury.

2 Institute de Biotechnologie, Domaine de Vilvert, 78350 Jouy-en-Josas, France.

56. Segregational instability of plasmids expressing staphyloccocal protein A

A. Warns' ${ }^{1}$ J. R. Stephenson ${ }^{1}$, and M. R. W. Brown ${ }^{2}$

'Biologic Division, PHLS Cama, Porton Down, Salisbury SP4 OJG.

${ }^{2}$ Department of Pharmaceutical Sciences, Aston University, Birmingham. 